\title{
Polysèmes
}

Revue d'études intertextuelles et intermédiales

\section{Absence et attentat : l'écriture de la défiguration dans Mao II de Don DeLillo}

\section{Florian Treguer}

\section{(2) OpenEdition}

Journals

\section{Édition électronique}

URL : http://journals.openedition.org/polysemes/1667

DOI : 10.4000/polysemes. 1667

ISSN : 2496-4212

Éditeur

SAIT

\section{Édition imprimée}

Date de publication : 1 janvier 2005

Pagination : 137-163

ISSN : 0999-4203

\section{Référence électronique}

Florian Treguer, "Absence et attentat : l'écriture de la défiguration dans Mao // de Don DeLillo »,

Polysèmes [En ligne], 7| 2005, mis en ligne le 01 janvier 2005, consulté le 03 mai 2019. URL : http:// journals.openedition.org/polysemes/1667 ; DOI : 10.4000/polysemes.1667

Ce document a été généré automatiquement le 3 mai 2019.

Polysèmes 


\title{
Absence et attentat : l'écriture de la défiguration dans Mao II de Don DeLillo
}

\author{
Florian Treguer
}

«La violence ne peut viser qu'un visage. » Emmanuel Lévinas ${ }^{1}$

À la parution de Mao II (1991), dixième roman du New-Yorkais Don DeLillo, la presse spécialisée s'est emparée de manière quasi unanime de l'extrait suivant, qu'elle a jugé bon de citer comme s'il résumait problématiquement l'enjeu majeur du texte: "There's a curious knot that binds novelists and terrorists. In the West we become famous effigies as our books lose the power to shape and influence. [...] Years ago I used to think it was possible for a novelist to alter the inner life of the culture. Now bomb-makers and gunmen have taken that territory. They make raids on human consciousness $»^{2}$. Ensuite, puisque ces phrases émanaient du personnage central, écrivain aussi réputé que secret, cette même presse a eu tôt fait de prêter ces propos directement à DeLillo lui-même, tout aussi connu pour son effacement. Par ailleurs, cette fascination pour la réalité agissante $\mathrm{du}$ « discours » terroriste, propre à éclipser l'impact de la fiction, n'épargnerait pas le romancier, comme en témoigne cette autre remarque du même personnage : « Beckett is the last writer to shape the way we think and see. After him, the major work involves midair explosions and crumbled buildings. This is the new tragic narrative » (157). Bien entendu, on sait d'une telle inscription en abyme du dire de l'écrivain qu'elle permet à DeLillo d'introduire du jeu, d'en modérer le propos dans ce dédoublement même, de le mettre à distance, comme entre guillemets. Pour autant, à bien y regarder, et contrairement à l'interprétation d'une critique toujours prompte à déplorer la perte d'influence de la littérature, il ne s'agit pas, en première analyse, de la simple reddition de l'écrivain face à l'éloquence inquestionnée (et dûment relayée par les médias) de l'acte terroriste. Bien plutôt, il est ici question de la confrontation de deux violences, d'un affrontement pour l'annexion puis la conservation d'un territoire (« that territory ») - en l'occurrence, un imaginaire de masse que le texte choisit de métaphoriser sous les espèces dramatiques d'un champ d'action, ou mieux, d'un théâtre des opérations ${ }^{3}$. De fait, 
entre terroristes et romanciers, la violence se retrouve bel et bien des deux côtés, qui cherche toujours à asseoir, selon des modalités distinctes et opposées (« alter the inner life » / « make raids»), une même mainmise sur la conscience collective («the power to shape and influence »).

\section{Alphabet et arsenal : la violence perdue du mot}

2 Ce thème d'une violence perdue au langage, qui lui aurait été d'une certaine façon consubstantielle et immanente, n'est pas vraiment nouveau chez DeLillo. Pour ne citer que deux précédents, on évoquera tout d'abord la violence du nom propre telle qu'elle se trouve littéralement scénographiée dans The Names (1982). Dans ce roman, le vecteur narratif est une quête archéologique des origines du nom, une tension vers ce momentcharnière et proprement mythique de l'émergence des premiers signes d'écriture. Au cœur de l'intrigue, sous-tendant la progression des protagonistes, le lecteur découvre une succession énigmatique de meurtres d'apparence rituelle. À travers deux enquêtes menées en parallèles, on apprend qu'ils seraient le fait d'une secte, les Abécédariens. La logique terroriste que ces derniers ont pu mettre en place se révèle des plus arbitraires. Elle consiste à faire coïncider les initiales d'une victime potentielle, nom et prénom, avec celles du nom du lieu où elle se trouve. Le cas de symétrie parfaite, lorsque concordent données patronymiques et toponymiques, entérine alors réciproquement le choix définitif de la victime et le lieu de son exécution - pour exemple, le meurtre de Michaelis Kalliambetsos à Mikro Kamini. De fait, il s'agit là d'une stratégie encore plus terrible que l'attentat aveugle car elle implique que les personnes assassinées ne sont pas, à proprement parler, innocentes. Elles sont littéralement victimes de leur nom autant qu'elles sont coupables, aux yeux de la secte, d'en être les dépositaires. Dans ce moment strictement paranoïaque, le nom parle, il s'affranchit de l'individu et marque une tension vers autre chose. On rejoint cette remarque de Gilles Deleuze selon laquelle le nom d'un individu met enjeu l'agencement collectif dont il est le produit et le signe (en l'espèce, un nœud de populations et de territoires). Il faut rappeler, à cet égard, que la violence sémantique de la secte prend pour point de départ la perte de sens dont souffriraient les noms propres aujourd'hui, le fait qu'ils existeraient confinés dans l'oubli de leur source prélinguistique. Les attentats auraient ainsi, pour leurs auteurs, cette fonction de rappeler le propre $\mathrm{du}$ nom, à savoir son histoire sous-jacente, les multiplicités (territoires, saisons, météores, etc.) qu'il subsume. Ils mimeraient surtout, dans leur violence meurtrière, la violence mythique et inaugurale, celle-là même qui a arraché l'alphabet à la géographie. Le but dernier d'une telle opération est alors de redonner du sang neuf aux noms (figurément un sens neuf), de leur communiquer métaphoriquement la portée effective de cette violence.

Plus généralement, The Names insiste sur l'idée d'une violence qui serait immanente à l'ensemble des noms propres. C'est ainsi à une même lumière qu'il nous faut lire l'étrange question que pose un des personnages: "If you think the name of the weapon is beautiful, are you implicated in the crime? $»^{4}$. L'œuvre revient régulièrement sur cette fascination trouble qui procède de la contemplation des noms d'armes, souligne leur beauté sinon leur grâce, comme il apparente implicitement l'alphabet à une manière d'arsenal. Plus particulièrement, elle interroge la manière dont le signifiant semble spécieusement épouser le signifié en esprit, et se mêler indissolublement à lui, dans ce que Saussure appelait l'image acoustique du mot. 


\section{La violence effective du langage}

Avec End Zone (1972), on franchit un pas de plus. Le langage est ici, à plusieurs reprises, défini comme seule instance d'une causalité «im-médiate ». Plus exactement, il lui est régulièrement associée une effectivité, quelque chose de cette qualité performative que J.L. Austin a reconnu à certains faits de langue, dans des conditions d'énonciation spécifiques. Parler devient le moyen de modifier directement l'ordre des choses. Et dans le monde à tendance entropique de End Zone, où le langage perd graduellement de sa signification, nommer revient à dégager une structure (" pattern ») à partir de l'informe et à s'inscrire, ce faisant, dans le sillage de l'acte divin, celui du logos onoma créateur. Le roman abonde en exemples où l'intention métaphorique ne vise jamais qu'à traduire l'intransitivité entre le mot et l'acte. Telle cette déclaration d'amour : "The words were ways of touching and made us want to speak with hands $»^{5}$. On retrouve un sens identique de ce raccourci dans la formule axiomatique, véritable clef de ce thème, avancée par le protagoniste et narrateur, Gary Harkness : «Words move the body into position. In time the position itself dictates events »(45). Cet enchaînement transitif («words » " position» - « events ») détermine bien deux temps et deux mouvements : l'action de mouvoir est directement attribuable au langage, l'événement à une certaine occupation de l'espace. Seulement, l'écriture vise ici clairement l'assimilation: un mot suffirait à faire advenir un fait dans le monde. La parole proférée libère ainsi des potentialités, elle est une action en devenir, une violence active susceptible d'ouvrir une brèche dans l'Histoire.

5 Comme fascinés, les romans de DeLillo reviennent régulièrement sur cette articulation singulière entre langage et événement, sur cette parole qui fait l'Histoire, semble la fabriquer dans une simple distribution spatiale de ses acteurs («[History] is also the placement of bodies. What men say is relevant only to the point at which language moves masses of people or a few momentous objects into significant juxtaposition. »(45). En particulier, l'œuvre de DeLillo résonne de cette parole-origine des grands mouvements collectifs, du mot d'ordre qui jette des millions de Chinois dans la Longue Marche, puis de la récitation du petit livre rouge qui instrumente la Révolution culturelle (Mao II), du slogan propagandiste qui architecture les colonnes nazifiées des rassemblements monumentaux de Nuremberg (White Noise, 1985, ou bien encore Running Dog, 1978), de la parole qui agenouille simultanément des millions de croyants dans le monde, les prostrant vers un même azimut (The Names), enfin du mot messianique qui réunit et unit, dans la répétition verbale, les corps votifs des grands mariages Moon - occasion d'une démultiplication de la puissance performative du «je vous marie »(prologue de Mao II). Cette parole-là meut et mobilise, fédère et fascine les masses (littéralement, les met en faisceau). En vectorisant ainsi sa propre force d'appel, en la canalisant puis en la dirigeant, elle est capable d'une incidence sur le cours des événements.

\section{L'itinéraire de Mao II : de l'écart à la fuite}

6 À défaut d'être foisonnante, l'intrigue de Mao II est toutefois complexe. Ramenée à la portion qui nous intéresse plus particulièrement, elle pourrait se résumer de la façon suivante. Écrivain aussi célèbre qu'invisible, que deux minces romans ont suffi à porter à une reconnaissance tant critique que publique, Bill Gray, personnage central de notre 
roman, s'est toujours tenu à l'écart des médias. Il vit reclus avec Scott Martineau, un jeune admirateur dévoué qu'il a recueilli et qui lui sert de secrétaire et de factotum, et Karen Janney, l'amie de Scott et ancienne adepte de la secte Moon, qui bien que « déprogrammée » (sic), cherche encore en tous sens les signes d'une vision messianique. Le récit commence au moment où cette retraite en tant que système trouve son point de rupture, atteint ce que la science appelle un seuil de criticité. À travers cette fuite volontaire (évocatrice du destin littéraire de J.D. Salinger ou de Thomas Pynchon), donnée comme condition fondatrice d'une écriture de l'écart et de la marge (où doit se lire en abyme la posture critique de Don DeLillo lui-même), Bill Gray a voulu mourir au monde. Son dernier portrait publié remonte à plus de trente ans, et cette absence soulignée du visage figure au mieux la mort de l'auteur, déserteur de la sphère publique dès lors que celle-ci le force à se mettre en scène, l'oblige au jeu de l'auto-représentation. Son troisième roman est achevé depuis deux ans mais, insatisfait, l'auteur ne cesse d'y apporter d'interminables corrections. Sa réclusion prolongée a fini, semble-t-il, par priver l'écrivain de ce monde qui donnait vie ainsi que sens à son langage (désormais neutralisé ou émoussé). Sur la base de cette représentation que le romancier ne maitrise plus ( I I no longer see myself in the language "[48]), à l'image aussi de ce dernier roman indéfiniment repris, objet de vains remaniements, Bill Gray dresse ce constat d'échec, qui se révèle le véritable point de départ de sa fuite en avant : "I'm sitting on a book that's dead» (48). À ce point, l'intrigue va faire se coïncider trois faits distincts : le renoncement à un anonymat à la fois pesant et menacé, l'abandon de la fiction, l'engagement politique. Traqué par les journalistes qu'attire le double fait de sa notoriété et de son silence, Bill Gray effectue sa sortie hors de sa tour d'ivoire, pour se frotter à ce monde qui l'entourait sans l'atteindre et plonger au cœur même de l'événement qui causera sa perte. Il accepte de poser pour Brita Nilsson, une photographe professionnelle. Ce premier pas dans le monde extérieur, cette concession à la pression médiatique, le précipite bientôt dans une logique événementielle qui va le conduire de New York à Djouniyé au Liban, via Londres et Athènes, afin d'obtenir d'un groupuscule terroriste maoïste qu'il libère un jeune et obscur poète suisse (Jean-Claude Julien), détenu en otage à Beyrouth. Sur les conseils de son éditeur, Bill Gray accepte d'apparaître en public. Mais de simple prête-voix pour l'écrivain captif (il doit lire quelques-uns de ses poèmes lors d'une conférence), son engagement se fait irrésistiblement plus personnel au point qu'il semble devenir le double de l'otage et projette finalement de prendre sa place. Bill Gray meurt au terme de cette entreprise quelque peu désespérée, solitaire, et somme toute mystérieuse.

\section{"So much has been disfigured in the name of symmetry »}

7 Cette remarque tirée d'Americana $a^{6}$, son premier roman, résume assez bien la stratégie adoptée par DeLillo pour Mao II : faire la démonstration formelle, sinon formaliste, d'un modèle de défiguration (au sens de négation du singulier). Dans son ensemble, ce roman est ainsi remarquable par son extrême souci de symétrie. Structurellement d'abord, il se compose de deux parties qui comprennent chacune un nombre identique de sept chapitres, qu'encadrent un prologue et un épilogue. À l'intérieur de cette construction en miroir, viennent ensuite se réfléchir les symétries propres au récit. Pour illustration, le roman s'ouvre sur le mariage collectif de 6500 couples, organisé par le Révérend Moon dans l'enceinte du Yankee Stadium de New York, pour se fermer sur l'évocation de noces 
tout aussi surprenantes : en pleine nuit, un cortège nuptial que précède un tank traverse joyeusement les rues de Beyrouth en guerre. Plus largement, la diégèse s'articule autour d'oppositions qui polarisent diversement sa thématique, distribuent aussi spatialement ses acteurs pour croiser, en les confrontant, leurs trajectoires signifiantes (celles de Bill Gray et de Karen Janney, par exemple). Au confinement forcené de la première partie répond ainsi, en deuxième partie, le mouvement incertain de la quête, comme à la mort figurée de l'écrivain succède la mort littéralisée de l'homme privé.

8 Au-delà des parallèles, il s'agit de bien circonscrire des zones d'empiétement et d'échange, ou pour reprendre un modèle cher à DeLillo (celui de la propagation épidémique), d'entre-contamination des milieux mis en présence par le texte. Le roman entretient notamment une confusion signifiante entre les villes de New York et de Beyrouth, ses deux pôles géographiques, qu'il rend alors immédiatement, intransitivement, coextensives l'une à l'autre, dans l'œil témoin de Karen. Au cœur de Manhattan outrancière, lieu de grande désorientation, ville « où rien n'est déplacé quand plus rien n'est à sa place » (« When there is enough out-of-placeness in the world, nothing is out of place » [22]), la misère et la détresse humaines qui affleurent aux abords de Tompkins Square ont perdu leur spécificité: «It's just like Beirut, it looks like Beirut »(146), peut alors s'exclamer ce personnage. Paradoxalement, le propre de la vision rapprochée est ici d'estomper les différences entre l'Occident et le Proche-Orient, en permettant au roman de renverser à l'envi les perspectives, de multiplier les raccords analogiques et de faire circuler librement les signes. Il n'est pas jusqu'aux personnages qui ne puissent sémiotiquement s'échanger, ni sémantiquement se valoir. Pour exemple : Charles Everson, l'éditeur de Bill Gray (qui incite ce dernier à l'engagement public), et George Haddad, le porte-parole du groupe terroriste libanais, que leur fonction désigne immédiatement comme intermédiaires et médiateurs, participent en définitive du même commerce trouble, s'accordant pour vendre la notoriété d'un écrivain contre le martyre potentiellement médiatique du second ( $\mathrm{I}$ want the famous novelist to address the suffering of the unknown poet » [99]).

Le lecteur comprend bientôt que ce réseau serré des correspondances (qui fonctionnent comme autant d'aiguillages de l'interprétation), des analogies savantes, des rappels symétriques comme des répétitions (à commencer par le titre : une toile d'Andy Warhol, deuxième d'une série de 27 sérigraphies) détermine finalement un système d'équivalence à l'intérieur duquel les objets du récit tendent progressivement à se neutraliser les uns les autres : lieux, péripéties, personnages, etc., rentrent tous désormais dans une logique de marché où s'échangent, pour s'annuler en définitive, leurs valeurs respectives. À terme, il ne s'agit pas tant de néant révélé, du rien vertigineux qui saisirait la conscience existentielle, que de nullité purement arithmétique - comme on parle du produit nul d'une opération - et de négation de la singularité. C'est cette identité des termes où tout est égal, se vaut, s'échange ou bien s'annule dans la symétrie et l'équation, qui régit l'économie violente de ce roman.

\section{Le sujet figuré de la violence}

$10 \mathrm{Au}$ centre de ce vaste système d'équivalences comme au cœur de ténèbres de ce roman, se tient l'otage, cagoulé et détenu dans une cave aveugle, sur lequel pèse une menace de mort constante. À l'évidence, sa position le définit comme une simple monnaie d'échange introduite dans un circuit marchand, appelée à rentrer virtuellement dans toutes sortes 
de tractations, ainsi que l'énonce froidement un des terroristes : «You sale this, you trade that. Always there are deals in the works. So with hostages. Like drugs, like weapons, like jewelry, like a Rolex or a BMW» (235). À partir du sort du jeune poète suisse (que sa citoyenneté va jusqu'à marquer d'un nouvel effet de neutralité symbolique), le roman développe ce qu'on appellera une fonction-otage paradigmatique, celle de "toujours valoir pour ", qu'il va progressivement étendre à tous les objets de son récit. Par définition, un otage n'a de valeur en soi ni fixe, ni propre, ni intrinsèque. Il est à tout instant de sa captivité un gage et un échange potentiel, la promesse d'un marché en devenir. L'acception du terme reflète bien cette tension duelle: «1. Personne livrée ou reçue comme garantie de l'exécution d'une promesse, d'un traité. 2. Personne que l'on arrête et détient comme gage pour obtenir ce que l'on exige. " (Dictionnaire Robert de la langue française). La vraie valeur de l'otage, toujours négociable, est en constant différé; plus loin, on dira même qu'il n'a fonctionnellement de valeur que dans cette attente. Dès l'instant où il vaut exactement une chose, il perd l'ouverture sémantique qui lui est propre et définitoire, pour n'être et ne valoir strictement que cette chose pour laquelle il s'échange et à laquelle désormais il se réduit : clôture de l'équivalence et mort symbolique de l'otage.

Outre de n'avoir alors de sens que dans la demeure (comme gage ou comme garantie) et dans l'horizon éventuel de la rançon, la figure de l'otage permet à DeLillo d'associer dans l'économie du roman, le sens définitif du texte à cette idée même du sursis et de la mort en suspens. La signification qu'il convient de donner à telle péripétie du roman demeure ainsi tributaire du sort central de l'otage, du règlement transactionnel dont il est l'objet, et s'indexe provisoirement sur son « omnivalence " paradigmatique. Le meilleur exemple de cette stratégie de l'atermoiement reste bien entendu l'itinéraire de Bill Gray que vectorise, à chaque étape et dans une logique de rapprochement constant, la perspective de l'otage à libérer. L'interprétation doit s'effectuer ici dans les deux sens : l'engagement et la démarche de Bill jusqu'à son sacrifice donnent une valeur et un prix à l'otage autant qu'ils reçoivent de lui, en retour, une justification et un sens ultimes. Dans l'intervalle, alors qu'il oscille entre ce qu'il est et ce qu'il vaut, que son identité intime est levée, hypothéquée, jusqu'à une libération qui ne viendra pas (et qui seule pourrait le défaire en tant que tel), l'otage figure au mieux la finalité dérobée du sens, de même qu'il métaphorise pour tout le texte la violence faite à la singularité.

\section{Violence de l'absence et attentat de l'écriture}

12 À partir du jeu d'oppositions qui architecturent l'espace sémantique de ce roman, j'ai choisi d'isoler une double tentative de mise en scène dialectique de cette violence de l'écriture. DeLillo déploie deux séries narratives convergentes: d'une part, l'opération terroriste (enlèvement et détention d'un otage), d'autre part, le travail d'imagination et d'écriture du romancier qui se fait " pour » et " en direction » de ce même otage, et vise à terme à rétablir son identité perdue. Au point d'intersection de ces deux séquences qu'organise un savant principe d'alternance, une logique confrontationnelle, le roman inscrit dans un parallèle signifiant le fait d'une double absence :

When you inflict punishment on someone who is not guilty, when you fill rooms with innocent victims, you begin to empty the world of meaning and erect a separate mental state, the mind consuming what's outside itself, replacing real things with plots and fictions. One fiction taking the world narrowly into itself, the other fiction pushing out toward the social order, trying to unfold it. (200) 
13 Dans l'amalgame des deux acceptions du mot "plot » en anglais, la mise en intrigue de l'otage (le mort en sursis, en suspens) est invariablement complice du complot: selon DeLillo, on retrouverait à l'œuvre dans le récit ou la conspiration, la même tension vers le vide, le même processus mortifère (« There is a tendency of plots to move toward death. [...] the idea of death is woven into the nature of every plot. A narrative plot no less than a conspiracy of armed men » peut-on lire dans Libra, autre roman de l'auteur $\left.{ }^{7}\right)$. En d'autres termes, dans la perspective du romancier qui écrit pour l'otage (l'autre absent, l'absence figurée, le visage invisible et nié), l'acte de mettre en fiction ("emplotment») la disparition ou l'absence au monde reviendrait à participer de la violence terroriste, en remplaçant un vide moralement irréductible, un innommable (le fait d'une violence unique, irreprésentable et muette) par la possibilité, le multiple, qu'engage et qu'implique inévitablement toute tentative fictionnelle. La représentation marquerait, en l'espèce, une trahison de l'intention. Effets croisés: comme le terroriste, l'écrivain épuise littéralement la réalité, la fait rentrer dans un processus d'exhaustion et de dévitalisation. Il remplace en les nommant, les choses réelles par des complots et des fictions (« replacing real things with plots and fictions »)

À ce titre, les médias rejoignent aussi les terroristes : tous deux produisent et promeuvent un double simulacral de l'otage, ajoutent au monde la menace d'une identité concurrentielle - entérinant ainsi cette peur de l'otage que le monde ait oublié son corps : « He had tumbled into the new culture, the system of world terror, and they'd given him a second self, an immortality, the spirit of Jean-Claude Julien. He was a digital mosaic in the processing grid, lines of ghostly type on microfilm. They were putting him together, storing his data in starfish satellites, bouncing his image off the moon. He saw himself floating to the far shores of space, past his own death and back again. But he sensed they'd forgotten his body by now. He was lost in the wavebands, one more code for the computer mesh, for the memory of crimes too pointless to be solved. »(112). Nouvelle économie de l'otage : le soi numérique est oubli et négation à terme du soi physique. La représentation se substitue ici avantageusement à la mortalité du corps. Cet échange de deux identités où, dans la non-coïncidence, l'image fait mortellement écran au corps, se veut être l'écho direct de ce deuxième soi (« a second self » : l'expression est identique à celle utilisée pour désigner l'otage cybernétique) que Bill Gray a le sentiment très net d'avoir « acquis » devant l'objectif de la photographe («I think I've grown a second self in the room. » 37)

\section{Le sens ôté de l'otage : l'écriture de la soustraction}

15 Si l'étymologie nous confirme bien que l'otage (comme l'anglais « hostage ») procède effectivement du mot "hôte », il rejoint sous la plume de DeLillo, et comme par une étrange coïncidence linguistique, l'idée même de soustraction : l'otage, est-il répété, est ici un sens ôté du monde. Sa détention prive le monde d'un fil supplémentaire de signification: "This poet you've snatched. His detention drains the world of one more thimble of meaning. » (200). Et si, pour le romancier, la prise d'otage apparait comme une manière de soustraction sémantique, il revient de plein droit à l'écriture de rétablir ou de restituer cette signification perdue pour le monde :

He was writing about the hostage to bring him back, to return a meaning that had

been lost to the world when they locked him in that room [...]. (200) 
16 au monde de l'otage. La question corollaire que pose alors un roman comme Mao II est celle, problématique, d'une écriture du retrait, de la défalcation, car "re-présenter» serait encore ici faire violence en épuisant la réalité, en lui retirant sa part d'innommable.

On le voit, la violence telle qu'on l'entend ici dans son rapport au langage s'oppose diamétralement à l'acception que lui donne le philosophe Éric Weil. Pour ce dernier, la violence est l'Autre du langage et se définit foncièrement dans son absence au discours, dans la radicalité du mutisme et de l'informulé; dès qu'elle trouve à s'exprimer par le langage, qu'elle accède à la parole, sa compréhension dans le discours commence de l'entamer, de la réduire et de la sublimer, car la violence pure est proprement indicible ${ }^{9}$. Dans Mao II, le renoncement à la fiction, à la logique de mise en intrigue (« emplotment »), figure un geste fondateur. Il a l'heur de déterminer l'ouverture d'un autre champ d'exploration de l'écriture, hors du représenté. Alors qu'elle tente de convoquer l'image de l'otage, l'écriture de Bill Gray renoue avec une inspiration essentielle, son geste mime à nouveau le travail d'une tension et d'une violence qu'il croyait perdues : « [...] feeling something familiar, something fallen into jeopardy, a law of language or nature, and he thought he could trace it line by line, the shattery tension, the thing he'd lost in the sand of his endless novel » (168).

ais l'écriture peut-elle évoquer la violence sans ajouter à cette violence, sans se constituer comme négation supplémentaire de cette absence, sans faire à son tour violence au sujet qu'elle ne peut s'empêcher de «défigurer ", de nier dans sa singularité et son intégrité ? Il faut ainsi pour l'auteur envisager, dans une sortie du récit, un autre mode de convocation de l'absence, une tentative d'écriture liminale, " au bord du vide ", qui fasse événement dans la possibilité laissée au langage d'inverser les termes de l'équation première: le sens de la violence (défiguration)/la violence du sens (refiguration). On est proche, en ces parages, de la dialectique développée par Maurice Blanchot quand il évoque la puissance de négation du mot, cet «assassinat différé » qu'est le langage qui nomme : « Le mot me donne ce qu'il signifie mais d'abord il le supprime. Pour que je puisse dire : cette femme, il faut que d'une manière ou d'une autre je lui retire sa réalité d'os et de chair, la rende absente et l'anéantisse. Le mot me donne l'être, mais il me le donne privé d'être. Il est l'absence de cet être, son néant, ce qui demeure de lui lorsqu'il a perdu l'être, c'est-à-dire le seul fait qu'il n'est pas $»^{10}$.

\section{La lettre au risque du vide}

19 C'est dans ce même sens qu'il faut tenter de présenter l'acte d'écriture dans Mao II. Celuici ne consiste nullement à combler l'absence (qui fonderait une nouvelle violence ajoutée) mais définit une tentative de restaurer l'otage, (l'Autre ou le Visage, peu importe, en définitive, le nom qu'on lui donne) dans l'absence même. Car c'est bien du vide, de l'obscurité de la cave ou de l'oubli de la cagoule, de cette circonstance toujours négative, que sa figure doit finalement surgir. Ce travail de l'écrivain n'est pas sans évoquer la restauration de tableaux, dans ce que l'approche peut avoir de périphérique, dans cette exigence commune d'un mouvement concentrique qui toujours resserre au plus près un centre absent, une trouée qu'il faut réduire. L'écriture doit ici s'aventurer et se mettre en jeu, se risquer dans un mouvement contraire d'avancée et de retrait, se soumettre, en d'autres termes, à la menace de bouleversement de cette "shattery tension " déjà évoquée (168). Elle entretient à dessein sa propre labilité comme elle se laisse miner par le

Polysèmes, 7 | 2005 
travail intestin du doute. L'enjeu qu'elle tente de saisir dans le même temps où elle se déploie, l'ouvre alors sur un "espace d'angoisse » familier, l'expose à la dérobade et au risque de la perte irréparable, corollaire d'une inspiration aussi fébrile que faillible: "There was something at stake in these sentences he wrote about the basement room. They held a pause, an anxious space he began to recognize. There's a danger in a sentence when it comes out right, a sense that these words almost did not make it to the page » (167).

Si la violence réside premièrement dans la négation de l'otage sans visage, l'écriture du visage (postulat de Mao II : il y a des visages lisibles et d'autres scriptibles) devient l'improbable comparution d'un sens qui se construit autour d'une absence pertinente, d'un trou noir, d'un bord de vide. Problème de cette sommation de paraître, de cette comparution hantée de l'absent (" to return a meaning that had been lost to the world »). Laurent Jenny nous rappelle en ce sens que « [1]a parole en effet ne trouve pas un monde tout constitué avant elle, et qu'elle se contenterait d'épeler. Tout autant elle produit ce monde en le sommant de paraître ${ }^{11}$. L'écriture n'a de sens que dans cette prise de risque, par ce pari qu'elle choisit d'énoncer de manière symptomatique par la bouche même de l'otage :

"[...]He tried to see his face in words, imagine what he looked like, skin and eyes and features, every aspect of that surface called a face, if we can say he has a face, if we believe there is actually something under the hood." (204-205)

\section{Une mise en scène de l'absence}

21 À son tour, dans le recueillement compassionnel, l'écrivain Bill Gray essaie d'imaginer la réclusion de l'otage, de voir et d'écrire son visage par une mobilisation de toutes ses facultés d'évocation : «Bill tried to see his face, hair, eye color, he saw room color, faded paint on the walls. He pictured precise objects, he made them briefly shine with immanence, a bowl for food, a spoon constructed out of thought, perception, memory, feeling, will and imagination » (154). Seul le travail d'écriture permet d'instrumenter une révélation immanente du visage. Il est ici l'unique moyen d'attenter à l'anonymat de la cagoule - le langage devenant la seule lumière susceptible de guider métaphoriquement le cheminement de la pensée jusque dans la cave obscure («[...] slowly building chains of thoughts, letting the words lead him into that basement room»160). Comme précédemment, l'objectif est de rendre visible par la seule puissance d'évocation des mots. À l'opposé, depuis la cave obscure qui l'enlève au monde, l'otage, un autre écrivain, désespère de ne pouvoir écrire pour donner corps et présence à ses pensées, les inscrire salutairement dans le monde sensible («He wanted paper and something to write with, some way to sustain a thought, place it in the world. »110).

Cette mise en scène de l'absence, de la vacance, se fait alors dans ce roman dans le passage répété de la vision à la visualisation. Le texte se construit exclusivement à partir de la perspective de l'otage dont il compense la cécité à la faveur de contrepoints visuels imaginaires. À défaut de voir, l'otage cagoulé est condamné à deviner les regards posés sur lui, à pressentir la présence regardante de celui qui le séquestre. À la vision proprement dite se substitue alors la visualisation, l'acte de rendre présent ce qui ne l'est pas, la présentification de l'invisible. C'est en ce sens qu'il faut interpréter, dans ce contexte précis, l'abus apparemment paradoxal de l'expression «to see ». Il est ainsi dit de l'otage : «He had to see his thoughts to keep them coming»(202). Visualiser, c'est 
créer, en l'espèce, un espace mental où inscrire et déployer réflexivement ses propres pensées. Ailleurs, pour "voir" son ravisseur, l'otage supplée au défaut de champ extérieur en donnant chair aux mots, en atteignant à cette image dénotative ou noétique du mot, qui est ici gage d'ouverture sur le monde perdu: «He tried to see his face in words », nous est-il encore donné de lire (204). En ce sens, la "figure» visée dans le langage serait cette force de mobilisation ou de convocation du mot, sa puissance à donner présence à l'éther de la langue («[make it] shine with immanence »). Jacques Derrida nous explique ainsi de ce processus de l'image noétique qu'il requiert l'éclipse ou du moins la dispense du mot : « Dans cette imagination du mot, dans cette représentation imaginaire du mot (Phantasie- vorstellung), nous n'avons plus besoin de l'événement empirique du mot. [...] Car si nous avons besoin de l'imagination du mot, du même coup nous nous passons du mot imaginé. L'imagination du mot, l'imaginé, l'être- imaginé du mot, son « image » n'est pas le mot (imaginé) » ${ }^{12}$.

\section{Le visage introuvable} visage de Bill Gray, autre écrivain - comme l'invisibilité du premier demeure indissociable, dans l'organisation symétrique du roman, de l'image plurielle de la masse anonyme. Deuxième crise de l'image : à travers Bill Gray, DeLillo explore l'autre visage absent et pose la question de la figure publique de l'artiste en regard de son œuvre ( $"$ The book disappears into the image of the writer » [71]), dans une société iconolâtre où la visibilité et l'exposition maximales sont devenues la norme, et la figure de l'invisible une transgression intolérable ainsi que l'objet paradoxal du plus vif désir («When a writer doesn't show his face, he becomes a local symptom of God's famous reluctance to appear. » [36]). Au début du roman, alors qu'il est traqué par les journalistes, l'écrivain caché est ainsi divinisé sur l'unique principe de son invisibilité au monde: «The writer who won't show his face is encroaching on holy turf. He's playing God's own trick » (37). Pour Bill Gray, l'artiste a dorénavant un devoir d'image à l'endroit de la société, il est devenu l'otage d'une image publique à laquelle il lui est demandé intempestivement de correspondre. Ultime transaction du récit: le livre s'échange contre le visage de son auteur. Le roman de Bill, non publié, sera finalement effacé, éclipsé, par la publication posthume des portraits pris par Brita («But the manuscript would sit, and word would travel, and the pictures would appear, [...] and word would build and spread, and the novel would stay right here, collecting aura and force, deepening old Bill's legend, undyingly » 224).

Enfin, on l'aura compris, la perte du visage, c'est aussi, dans ce roman, l'aura dévoyée. On se souvient que chez Walter Benjamin, l'aura s'apparentait à « l'apparition d'un lointain si 
proche soit-il », qu'elle était le marqueur d'une unicité sacrée et non-reproductible. Ici, dans une conception diamétralement opposée, elle est devenue le signe de la chose reproduite, voire l'indicateur d'intensité de sa reproduction («Everything seeks its own heightened version. Or put it this way. Nothing happens until it's consumed. Or put it this way. Nature has given way to aura » 44). Pour cette œuvre qui convoque Andy Warhol ${ }^{13}$ à maintes reprises (jusqu'à son titre), qui multiplie aussi les effets de répétitions sérielles et de simulacre, il existe une équation implicite entre ce que représente l'aura (conçue comme "une singulière trame de temps et d'espace») et l'inédit du visage, son événement.

\section{Lire le singulier ou la différence " envisagée »}

Devant ce système d'équivalence, ce circuit fermé des valeurs comme cette consommation auratique du monde, se pose alors la question de la différence. Une différence qu'on identifiera à un principe de l'unique, car soutirer ici la plus petite différence qui soit, c'est encore défier l'empire de l'identitaire, résister à l'impersonnalité de la troisième personne. De fait, contre la défiguration, DeLillo fait essentiellement jouer une différence vive sinon vitale : le visage - en tant qu'il est une puissance d'affirmation et de singularité, une altérité incessible qu'il faut extraire de la foule dévisageante, retrouver aussi sous la négation de la cagoule opaque. On pourrait dire du rétablissement de la différence qu'il se révèle la tension sous-jacente qui travaille le sens intime de l'écriture.

Posture emblématique du personnage de Karen face au spectacle de la masse meurtrière de Sheffield (49-51): elle s'efforce de "lire des visages» dans l'amas monstrueux et compact des corps. Son regard défait littéralement la foule martyre, tente de lui rendre une visagéité, en libérant l'unique de la personne dans le phénomène de la rencontre - dès qu'il est possible d'isoler la singularité d'un seul sujet, la masse ne peut demeurer complètement : l'altérité absolue du visage possède, en l'espèce, une vertu disruptive. À partir d'un arrêt sur image, Karen va alors briser progressivement l'identité de cette masse en la fragmentant, en la scrutant comme un tableau dans toutes ses parties, dans sa composition même. Devant cette scène de passion (« it is like a religious painting, the scene [...] is composed and balanced and filled with people suffering »), l'action de Karen tient alors du personnage biblique de Véronique (la « vera iconica») qui, dans le creux de son voile, recueille le visage du Christ au calvaire, arrache son empreinte à la souffrance. Surtout, les visages deviennent, à ce point, lisibles («In people’s faces she sees the hopelessness of knowing »). La lecture est d'ailleurs à double sens : les visages sont ici lisibles et lus («to read faces») autant qu'ils expriment et donnent à lire (celui de tel homme : « his face is reading desperation »).

Dans ce roman, le lecteur est ainsi convié à lire les visages, à les recevoir aussi comme des masques de douleur (torturés, grimaçants, convulsés) - comme l'affirme de façon programmatique le personnage de Brita : «We ail try to read faces » (26). Pareille fiction nous dit alors diversement qu'il y a des visages lisibles et des visages scriptibles, qu'audelà des échanges à produit nul, il existe une économie du singulier, du don ou de l'abandon. On le sait depuis Lévinas, le visage ne peut s'échanger car il ne fait pas signe (il est, ne signalant que soi et sujectivité absolue), mais il définit ce lieu d'affleurement de l'être comme totalité visible. Il ne signifie pas, ni n'incarne, ni ne revêt. Il est immédiatement et irréductiblement expression de la personne, unité originelle du regard 
et de la parole. Il y a ainsi une évidence épiphanique du visage : son apparition est une première violence, un événement qui « déchire le sensible $»^{14}$.

\section{Le visage visé de l'écriture}

À l'omnivalence foncière de l'otage, l'écriture tente ainsi de répondre par l'unique du visage. Jacques Derrida note ainsi : "Seul un visage peut arrêter la violence car seul il peut la provoquer $»^{15}$. Si les terroristes, à travers la détention de l'otage sans visage, affichent un projet de défiguration (à savoir priver le monde d'une signification inédite et irremplaçable), ce roman assigne à la littérature une mission de refiguration, dans le recouvrement du sens perdu du visage (« to return a meaning that had been lost to the world »). Écrire en l'espèce contre l'afiguré de la masse (absence ou perte du singulier) et le défiguré de faction terroriste (négation violente du singulier). Rappelons que c'est d'ailleurs à partir de la relation otage-geôlier, que le roman pose la question d'un visage devenu introuvable : « [...] he tried to see face in words, imagine what he looked like, skin and eyes and features, every aspect of that surface called a face, if we can say he has a face, if we believe there is actually something under the hood.» (204-205). Le sens ultime de l'écriture ne tiendrait ici, en dernière analyse, qu'à ce geste de retrouver l'être du singulier sous les espèces dénaturantes du neutre. Telle pourrait être la tâche que Mao II assigne à la littérature, celle d'écrire des visages, de produire, autrement dit, des écarts différentiels, de conquérir ou rétablir le singulier dans un règne du même identitaire.

Dans sa préface à La Parole singulière de Laurent Jenny, Jean Starobinski explique que «le travail de singularisation laissera sa trace dans un objet, produira des structures (des configurations) mais c'est en vue d'autre chose: l'apparition d'un sens, l'éveil à des présences, fussent-elles passagères, intermittentes. [...] En liant le figural à la notion d'événement, peu s'en faut [...] que Laurent Jenny n'évoque la venue d'un visage. Mais il ne le fait pas, car c'eût été déjà trop dire, et en même temps délimiter à l'excès l'événement, priver la parole de son pouvoir propre, qui est de figurer sans prendre figure $»^{16}$. Le visage tel qu'il apparait dans Mao II est bien cette présence visée, entrevue comme par intermittence et se dérobant toujours, mais qui vectorise néanmoins le sens de l'écriture. Le visage comme événement constitue ainsi un dessaisissement, selon le mot de JeanFrançois Lyotard ${ }^{17}$, il détermine un sens qu'on pourrait qualifier de figural en ceci qu'il ne se laisse pas réduire à la circonstance de son apparition. Comme l'écrit justement Jean Bessière dans La Littérature et sa rhétorique: "Le visible - le visage - peut devenir une manière d'invisible; il peut transformer sa propre évidence dans la mise en face d'un masque et d'une totalité. Un masque : comme le visage qui ne relève ni de la connaissance ni de l'imaginaire ; totalité : comme le visage qui est son propre tout et ce qui fait face ${ }^{18}$. À travers la figuration du visage, l'écriture de DeLillo mime le projet et l'horizon de sens qu'elle se donne. En d'autres termes, le visage est ici à l'écriture sa propre violence, cet Autre irréductible qu'elle vise et sur lequel elle butte, et qui la justifie pourtant dans cette buttée même.

\section{Pour conclure}

31 Il reviendra en définitive au personnage de Brita Nilsson, la photographe, d'accomplir ce geste de dévoilement (et de rétablissement) du visage, dans le cours de l'épilogue - à ce déplacement près qu'elle s'en prend à l'identité masquée d'un des terroristes : «On an 
impulse she walks over to the boy at the door and removes his hood. Lifts it off his head and drops it on the floor. [...] And takes two steps back and snaps his picture » (236). On songe alors à ce que Walter Benjamin a écrit de la photographie, qu'elle est stricto sensu cet « art de démasquer $»^{19}$. C'est d'ailleurs, en un sens, la leçon de ce texte qui consigne la faillite plurielle de l'écriture, supplantée par l'efficace immédiate de l'image. Au terme du roman, l'otage sans visage devient ce vide, ce point d'effondrement où semble s'abîmer le récit tout entier.

Transaction symbolique ratée, la fiction ne peut s'échanger contre de la réalité: le "plot» terroriste annule le «plot» du romancier, ainsi que son illusoire volonté de se prolonger dans le monde ( One fiction taking the world narrowly into itself, the other fiction pushing out toward the social order, trying to unfold it » [200]). Finalement, la mort de Bill Gray vient se substituer à celle sans cesse différée de l'otage, son double à plus d'un titre, qui sera comme il se doit revendu (on ne sait à quel prix, on ne sait contre quoi) à une milice locale. La relation des derniers jours de Bill Gray, en transit vers un Liban qu'il n'atteindra pas, évite tout pathos tragique par la grâce paradoxale de cet événement où la singularité d'une trajectoire épouse l'indéfini d'une voix : dans le cours du roman, l'écrivain est progressivement devenu le support d'une douleur archétypique, évoquée à plusieurs reprises. D'un élancement profond et régulier que Bill ressent au côté droit (des suites d'un accident à Athènes où il s'est fait renversé par une voiture), le texte nous a déjà dit ainsi : «Sometimes a pain feels familiar even as it hits you for the first time. Certain conditions seem to speak out of some collective history of pain » (196). Tandis qu'il meurt, tout se mêle alors dans l'esprit de Bill Gray: ses souvenirs d'enfance, l'obsession douloureuse de l'otage, la pensée de la mort imminente et l'impuissance à écrire. Jusqu'au bout, il aura pourtant écrit obstinément sur le jeune poète, à tel point que le lecteur peut légitimement se demander si, à la faveur d'une transgression des niveaux de texte, par la grâce d'une métalepse audacieuse, les épisodes de la cave aveugle, ceux écrits du point de vue de l'otage, ne doivent pas en définitive lui être attribués.

\section{BIBLIOGRAPHIE}

Benjamin, Walter. «Petite histoire de la photographie ». In Essais 1 (1931). Trad. Maurice de Gandillac. Paris : Editions Denoël-Gonthier, 1983.

Bessière, Jean. La Littérature et sa rhétorique : La banalité dans le littéraire au XXe siècle. Paris : PUF, coll. « L'interrogation philosophique », 1999.

Blanchot, Maurice. La Part du feu. Paris : Gallimard, 1949.

Buci-Glucksmann, Christine. «La disparition du visage en art » in Revue des Sciences Humaines, $\mathrm{n}$

- 243, juillet-septembre 1996 : 131-139.

DeLillo, Don. Mao II. New York \& London: Viking Penguin, 1991.

DeLillo, Don. Americana. New York \& London: Penguin Books, 1971. 
DeLillo, Don. End Zone. 1972. London \& New York: Penguin Books, Contemporary American Fiction, 1986.

DeLillo, Don. Running Dog. 1978. New York: Vintage Books, Vintage Contemporaries, 1989.

DeLillo, Don. The Names. 1982. New York: Vintage Books, Vintage Contemporaries, 1989.

DeLillo, Don. White Noise. 1985. New York: Penguin Books, Contemporary American Fiction, 1986.

DeLillo, Don. Libra. New York: Viking Penguin, 1988.

Derrida, Jacques. « Violence et métaphysique : Essai sur la pensée d'Emmanuel Lévinas ». in L'Écriture et la différence. Paris : Éditions du Seuil, 1967, 117-228.

Derrida, Jacques. La Voix et phénomène. Paris : PUF, coll. « Quadrige », 1967.

Jenny, Laurent. La Parole singulière. Paris : Belin, 1990.

Lévinas, Emmanuel. Totalité et Infini : Essai sur l'extériorité. Paris : Fayard, coll. « Biblio Essais », 1971.

Lévinas, Emmanuel. Éthique et Infini. Paris : Fayard, coll. « Biblio Essais », 1982.

Lyotard, Jean-François. Discours, Figures. Paris : Klincksieck, 1971.

Tabbi, J. « From the Sublime to the Beautiful to the Political : Don DeLillo at Midcareer ». In Postmodern Sublime, Technology and American Writing from Mailer to Cyberpunk. Ithaca/London: Cornell UP, 1995.

Weil, Éric. Logique de la Philosophie. Paris : Vrin, 1950.

\section{NOTES}

1. Emmanuel Lévinas, Totalité et Infini : Essai sur l'extériorité, Paris, Fayard, coll. « Biblio Essais », 1971, 216.

2. Don DeLillo, Mao II, New York \& London, Viking Penguin, 1991, 41.

3. On peut lire également en ce sens : «What terrorists gain, novelists lose. The degree to which they influence mass consciousness is the extent of our decline as shapers of sensibility and thought. The danger they represent equals our own failure to be dangerous. » (157)

4. The Names (1982), New York, Vintage Books, Vintage Contemporaries, 1989, 118.

5. End Zone (1972), London \& New York, Penguin Books, Contemporary American Fiction, 1986, 217.

6. Don DeLillo, Americana, New York \& London, Penguin Books, 1971, 326.

7. Don DeLillo, Libra, New York, Viking Penguin, 1988, 221.

8. Joseph Tabbi propose une lecture de ce passage (et de l'enjeu croisé qu'il représente) à la lumière des catégories du sublime établies par Thomas Weiskel (The Romantic Sublime, 1976): «The mind that alternately empties the world of meaning and consumes meanings outside itself repeats a familiar pattern in the discourse of the sublime - specifically, the alternation between metaphorical and metonymical poles in Weiskel's analytic. In the first, metaphorical mode, 'the absence of determinate meaning becomes significant' and the mind 'resolves the breakdown of discourse by substitution,' in effect setting up a separate mental state of its own. 'The other mode of the sublime may be called metonymical. Overwhelmed by meaning, the mind recovers by displacing its excess of signified into a dimension of contiguity which may be spatial or temporal' (Weiskel 28, 29)» («From the Sublime to the Beautiful to the Political: Don DeLillo at 
Midcareer ", in Postmodern Sublime. Technology and American Writing from Mailer to Cyberpunk, Ithaca \& London, Cornell UP, 1995, 205).

9. Éric Weil, Logique de la philosophie, Paris, Vrin, 1950, 56-58.

10. Maurice Blanchot, La Part du feu, Paris, Gallimard, 1949, 312.

11. Laurent Jenny, La Parole singulière, Paris, Éditions Belin, 1990, 19.

12. Jacques Derrida, La Voix et le phénomène, Paris, PUF, coll. « Quadrige », 1967, 48.

13. Dans le champ de la représentation, ce thème de la disparition du visage doit être rapproché de l'exploitation que ce roman fait de l'œuvre dite postauratique d'Andy Warhol. Le choix d'une telle figuration peut alors se comprendre dans le sens de cette remarque de Christine BuciGlucksmann : «À force de violenter les visages, de les déformer, de les effacer, de les mettre en série ou en spectres, il ne reste plus que des têtes sans visage ou des métaphores plus ou moins ironiques du portrait. » (« La disparition du visage en art » in Revue des Sciences Humaines, $\mathrm{n}^{\circ} 243$, juillet-septembre 1996, 131).

14. Emmanuel Lévinas, Éthique et Infini, Paris, Fayard, coll. « Biblio Essais », 1982, 75.

15. Jacques Derrida, «Violence et métaphysique : Essai sur la pensée d'Emmanuel Lévinas » in L'Écriture et la différence, Paris, Editions du Seuil, 1967, 218.

16. Préface à La Parole singulière, Paris, Belin, 1990, 5-8.

17. Jean-François Lyotard, Discours, Figures, Paris, Klincksieck, 1971, 22-23.

18. Jean Bessière, La Littérature et sa rhétorique : La banalité dans le littéraire au $X X^{e}$ siècle, Paris, PUF, coll. «L'interrogation philosophique », 1999, 63.

19. Walter Benjamin, «Petite histoire de la photographie » in Essais 1 (1931), Trad. Maurice de Gandillac, Paris, Editions Denoël-Gonthier, 1983, 166.

INDEX

oeuvrecitee End Zone, Mao II, Names (The) 